\title{
Screening of differentially expressed genes induced by water-soluble extracts from pollen during honeybee caste determination
}

\author{
B. Zhou ${ }^{1}$, M.H. Ye ${ }^{2}$, K. Zhang ${ }^{2}$, Y.W. Wu ${ }^{1}$ and J.T. Ding ${ }^{2}$ \\ Yangzhou University, \\ ${ }^{1}$ College of Animal Science and Technology, \\ ${ }^{2}$ College of Bioscience and Biotechnology \\ Yangzhou, 225009, P.R. China
}

(Received 10 June 2009; revised version 8 February 2010; accepted 9 May 2010)

\begin{abstract}
In order to screen differentially expressed genes during caste differentiation induced by pollen, larvae of Apis mellifer ligustica were developed in an incubator under controlled temperature and relative humidity until emergence. Worker bee morphology was induced by addition of water-soluble extracts from pollen in larval diets. After all of the developmental periods, the external characters and developmental degree of ovaries were evaluated to confirm the caste of new adults. Transcripts from larvae of developing queens and workers were profiled by differential-display RT-PCR analysis and differentially expressed fragments were reconfirmed by dot-blot assay. Our results showed that caste differentiation was triggered by the intake of pollen extracts. Six transcript-derived fragments were isolated and three of them are reported for the first time. We conclude that differential display is a feasible approach to identifying differentially expressed genes. The identification of up-regulated caste-differentiation-related genes provided interesting clues about the activation of biochemical steps relevant to this progress.
\end{abstract}

KEY WORDS: honeybee, differential display, gene expression, pollen, caste differentiation

\footnotetext{
${ }^{3}$ Corresponding author: e-mail: mhye@yzu.edu.cn
} 


\section{INTRODUCTION}

The nourishment received by female honeybee larvae determines their differentiation into queens or workers (Wheeler, 1986; Mike et al., 2003). This developmental switch depends not on genotypic differences but on the differential expression of entire suites of genes involved with larval fate (Jay et al., 2001). To date, the identity of differentially expressed genes associated with queenworker caste determination in larvae has been investigated in the honeybee, and groups of related genes have been identified (Evans et al., 2000; Hepperle et al., 2001; Piulachs et al., 2003; Guidugli et al., 2004; Grozinger et al., 2007). It is still not understood, however, how differential nutrition is linked to gene expression and how dietary changes alter pathways that modify the development of genetically identical female larvae. In previous research, larvae were collected directly from the hive or transferred to an incubator before treatment. All of the detected differentially expressed genes gave us a complete profile of caste differentiation. In order to select genes that were expressed differentially due to pollen components, we decided to evaluate the function of water-soluble pollen extracts on larval development in the presence of royal jelly under laboratory conditions- Worker bees were induced by exposure to water-soluble extracts of pollen at early larval stage. Differentially expressed fragments were screened by non-targeted differential-display reverse transcription PCR (DDRT-PCR) analysis and re-confirmed by dot-blot assay. Considering their putative relationship to caste differentiation, genes up-regulated in developing worker bee larvae were useful in clarifying the timing and nature of genetic events during this process and may be considered of interest for further studies.

\section{MATERIAL AND METHODS}

\section{Rearing of bee larvae}

Larvae of Apis mellifer ligustica were collected in the College of Animal Science and Technology, Yangzhou University (China). We put an empty comb into a healthy colony. About 4 to $6 \mathrm{~h}$ after the queen laid the eggs, we removed the comb and placed it in the upper honey drawer to develop larvae. When larvae were $12 \mathrm{~h}$ old we transferred them to aseptic culture dishes with the help of a plastic grafting tool. Artificial diets were spread evenly on the bottom of culture dishes in an approximately $0.5 \mathrm{~cm}$ deep layer. Larvae were developed in an incubator (HSX-160B, Shanghai Fuma Laboratory Equipment Co., LTD) at a relative humidity of $85 \pm 5 \%$ and a temperature of $35.8 \pm 0.1^{\circ} \mathrm{C}$. 


\section{Preparation of water-soluble extracts from pollen}

Lyophilized Camellia pollen of known weight was stored in a jar at $-70^{\circ} \mathrm{C}$ for at least $24 \mathrm{~h}$. Sterile distilled water, pre-warmed to $75^{\circ} \mathrm{C}$, in an amount double the weight of the pollen, was poured into the jar and the mixture was stirred quickly with a glass rod to destroy the cell wall of pollen. The supernatant was collected after centrifugation at $12,000 \mathrm{~g}$ for $15 \mathrm{~min}$ at $4^{\circ} \mathrm{C}$, filtration through aseptic Whatman No.41 filter paper, and stored at $4^{\circ} \mathrm{C}$.

\section{Composition of artificial diets}

Larvae were distributed randomly into two feeding groups with different artificial diets with 40 larvae per group. Fresh royal jelly was collected from the 48-72 h old queen larvae reared in a healthy colony. Mature honey (Black Locust), containing 20\% water, was acquired from Yangzhou Tongren Bioengineering Co., LTD. The ingredients of daily diets (Table 1) were mixed thoroughly with a superfine homogenizer (F6/10, FLUKO, Germany) and prewarmed to $35^{\circ} \mathrm{C}$ before use.

Table 1. Compositions of artificial diets for larvae in different groups, weight $\%$

\begin{tabular}{lccccc}
\hline Group & Royal jelly & Honey & $\mathrm{H}_{2} \mathrm{O}$ & Pollen extracts & Water content \\
\hline $\mathrm{Q}$ & 48 & 16 & 36 & - & 69.60 \\
W & 48 & 16 & - & 36 & 68.88 \\
\hline
\end{tabular}

$\mathrm{Q}$ and $\mathrm{W}$ - larvae will develop to be queens and workers, respectively

\section{Larval weight records}

Larval weights were recorded at larval stages of $2 \mathrm{~d}(36 \mathrm{~h}), 3 \mathrm{~d}(60 \mathrm{~h}), 4 \mathrm{~d}$ $(84 \mathrm{~h}), 5 \mathrm{~d}(108 \mathrm{~h})$ and $6 \mathrm{~d}(132 \mathrm{~h})$, respectively. In order to reduce the adverse effect of weighing on larval development, larvae in each group were assigned randomly and evenly into two sub-groups and weighed alternately. Each larva in the sub-group that was being weighed on a given day was rinsed quickly by drops of sterile distilled water pre-warmed to $35^{\circ} \mathrm{C}$ to remove the diet adhering to its body and then was placed on aseptic filter paper to absorb the remaining water. Larvae in the other sub-group scheduled to be weighed the next day were transferred directly to new aseptic culture dishes with fresh diet spread in them. When larvae were observed to spin they were cleaned as described above and put on dry aseptic filter papers, allowing them to develop in the incubator until emergence. The developmental pattern of larvae was fitted by a nonlinear method based on the recorded larval weights and the corresponding equations were compared among different groups. 


\section{Statistical analysis}

Statistical analyses were performed using the one-way ANOVA procedure of SAS (SAS Inst., Inc. Cary, NC). When a statistical significance was detected $(\mathrm{P}<0.05)$, comparisons between means were carried out using Duncan's multiplerange test.

\section{Preparation of ovarian cryosections from new adults}

The whole body was frozen rapidly to $-30^{\circ} \mathrm{C}$ and embedded at the optimum temperature in a cutting compound. Frozen sections of the abdomen were prepared in a cryostat (Leica cm1900, Germany) and observed directly without being stained. The number of ovarian tubules was compared among specimens from different groups.

\section{Differential display of $m R N A$}

There were about 2 x 20 extra larvae developed under the same nutritional and environmental conditions as larvae in groups $\mathrm{Q}$ and $\mathrm{W}$, which were used for the genetic analyses. At larval stage of 60,84 and $108 \mathrm{~h}$, six larvae were used to extract total RNA separately. Total RNA $(200 \mu \mathrm{g})$ was prepared using the Trizol reagent (Invitrogen) and transcribed to three cDNA pools using three singleanchored primers, 5'- AAGCTTTTTTTTTTN (N was A, C or G) -3', and M-MLV reverse transcriptase (Promega) according to the manufacturer's instructions. One $\mu 1$ cDNA was added to the $25-\mu 1$ PCR mixture containing $2.5 \mathrm{mM}$ dNTPs, $10 \mu \mathrm{M}$ 5' 10-mer random deoxyoligonucleotide primer (No.226: 5'-TCGGTCATAG-3', No.228: 5'- TACGTAAGCG-3', No.234: 5'- GATCTG ACAC-3', No.235: 5'-GATCTCAGAC-3', No.240: 5'- GATCTCACTG-3') synthesized by Ubiogene Engineering Co., Ltd. Beijing (China), $10 \mu \mathrm{M}$ 3' single-anchored primer, and 1.5U Taq polymerase (Promega). Denaturing $\left(4 \mathrm{~min}\right.$ at $\left.94^{\circ} \mathrm{C}\right)$ was followed by 40 cycles of $30 \mathrm{~s}$ at $94^{\circ} \mathrm{C}, 90 \mathrm{~s}$ at $40^{\circ} \mathrm{C}$ and $50 \mathrm{~s}$ at $72^{\circ} \mathrm{C}$, with a final elongation step at $72^{\circ} \mathrm{C}$ for $10 \mathrm{~min}$. Amplicons were then size-fractionated by electrophoresis in $12 \%$ polyacrylamide gels and bands were viewed after silver staining.

Differentially expressed DNA fragments were excised from the gels, $50 \mu 1$ of sterile water were added, and incubated at $100^{\circ} \mathrm{C}$ for $5 \mathrm{~min}$. The eluted DNA was re-amplified in a $25 \mu \mathrm{l} \mathrm{PCR} \mathrm{mixture} \mathrm{using} \mathrm{the} \mathrm{same} \mathrm{pair} \mathrm{of} \mathrm{primers} \mathrm{used}$ in the differential display. PCR conditions were the same as described above. The confirmed differentially expressed fragments were ligated with the pMD-19T vector (Takara, Japan) and transferred into E. coli. strain DH5 $\alpha$. Confirmed blue positive clones were sequenced afterwards. 


\section{Dot-blot assay}

Specific primers were designed using primer 5.0 according to the sequence information of differentially expressed fragments. Total RNA from larvae was used as the template and RT-PCR was carried out to produce specific probes with digoxigenin-labeled dUTP. The cDNAs prepared with total RNA from larvae were dotted onto positive-charged nylon membranes. Dot-blot hybridization was conducted according to the manufacturer's instructions (Innogent Co., Ltd. Shenzhen, China).

\section{RESULTS}

Comparison of growth development. Statistical analysis showed that larvae in group Q always had significantly higher weights than larvae in group $\mathrm{W}$ during the entire larval stage $(\mathrm{P}<0.05)$. This advantage in weight was maintained until new adults emerged (Figure 1).

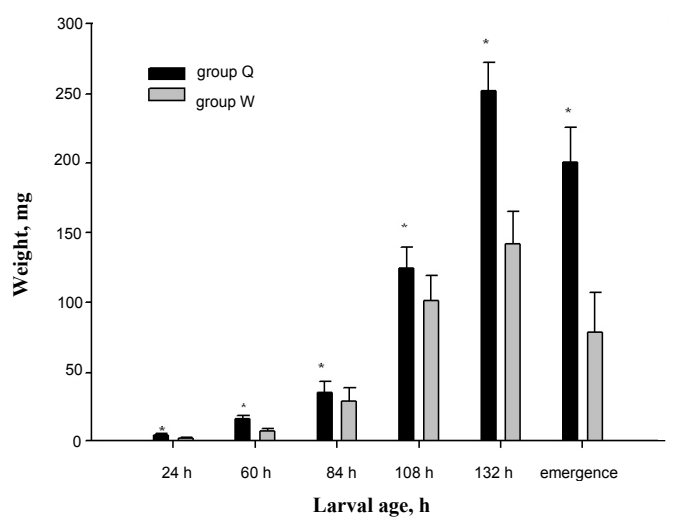

Figure 1. Comparison of larval weight at different developmental stages

Equations for estimation of larval weights at specific stages were:

$$
y=1.7428 e^{1.0508 x}\left(R^{2}=0.9934\right) \text { and } y=0.504 e^{1.3341 x}\left(R^{2}=0.9995\right)
$$

for larvae in groups Q and W, respectively. When corresponding data obtained from larvae developed under natural conditions (Yu et al., 2001) were compared 
with larval weights from the two tested groups, we found that the growth curve for larvae in group Q paralleled that of developing queen bees in the colony, while larvae in group $\mathrm{W}$ were similar to those of worker bees developed under natural conditions (Figures 2 and 3).

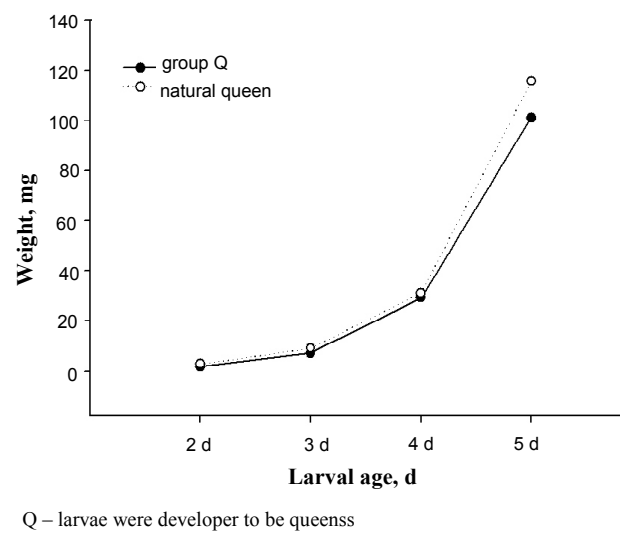

Figure 2. Comparison of weight at different stages between larwae developed in group Q and natural condition

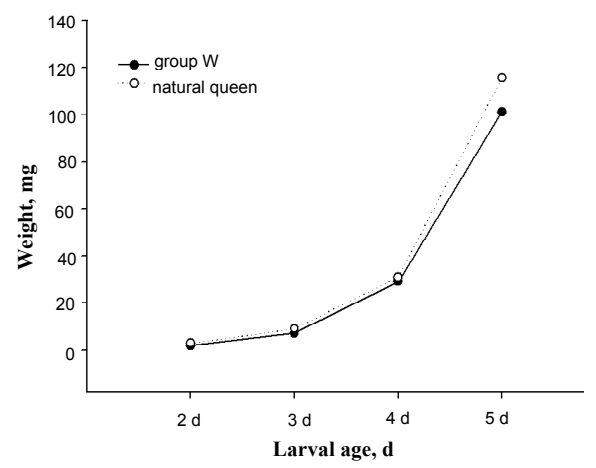

W - larvae were developer to be workers

Figure 3. Comparison of weight at different stages between larwae developed in group W and natural condition

Note: Weight at emergence was recorded at the exact time when a new adult emerged. 


\section{Comparison of developmental duration}

At the beginning of our experiment, the sample number in each tested group was 40 . There were still more than 38 larvae left in each group at the end of larval stage. Peak mortality occurred at the early (2-3 days) pupal stage and consequently resulted in a significantly lower emergence rate, 65\% (26/40) and 70\% (28/40) for larvae in groups $\mathrm{Q}$ and $\mathrm{W}$, respectively. We concluded that environmental factors had critical effects on pupae and the detailed environmental needs of pupae were worthy of further investigation.

Under artificial conditions we noticed that when a larva began to spin yellow substances around it, a loss in weight always occurred, which helped us to determine that it had stopped taking in food and had entered the pupal stage. The average developmental periods of larvae and pupae in the two tested groups were consistent with the corresponding data from developing queens and workers under natural conditions (Table 2). Since the only difference between larvae in the two tested groups was the existence of pollen extracts in the diets for larvae in group $\mathrm{W}$, we concluded that water-soluble ingredients from pollen inhibited the growth of larvae in group $\mathrm{W}$, i.e. significantly reduced larval weight and prolonged development.

Table 2. The average developmental periods for honeybees developed in different conditions, $\mathrm{h}$

\begin{tabular}{lccc}
\hline Item & Unsealed larval stage & Sealed pupal stage & Total \\
\hline Group Q & $115 \pm 2.13$ & $221 \pm 5.16$ & $336 \pm 4.73$ \\
Natural Q* & $116 \sim 123$ & $218 \sim 234$ & $334 \sim 357$ \\
Group W & $128 \pm 3.56$ & $380 \pm 7.83$ & $508 \pm 6.54$ \\
Natural W* & $124 \sim 130$ & $354 \sim 371$ & $478 \sim 501$ \\
\hline Q and W - larvae were developed to be queens and workers in laboratory. Q* and W* - queens and \\
worker bees were reared in the natural colony (data from the previous reports (Yu et al., 2001)
\end{tabular}

Comparison of external features. We found that the new adults that emerged from groups $\mathrm{Q}$ and $\mathrm{W}$ had typical features of queen and worker bees, respectively (Table 3). The highly developed ovary and underdeveloped wings for adults in group Q were consistent with the unique role queen bees play in the colony. External features reconfirmed that adults from group Q and group $\mathrm{W}$ represented two totally different castes. 
Table 3. Comparisons of external features between new adults from different groups

\begin{tabular}{|c|c|c|}
\hline Item & Group Q & Group W \\
\hline Head & heart-type & triangular shape \\
\hline Abdomen & long and thin, long conical shape & short and stout, conical shape \\
\hline Stinger & small, having barb & apparent, no barb \\
\hline Wings & half the length of abdomen & a little shorter than the length of abdomen \\
\hline Girdle bands & almost invisible & apparent \\
\hline Breast & wide & narrow \\
\hline Body & yellow, barely covered with villi & $\begin{array}{l}\text { dark brown, head and breast covered with } \\
\text { thick villi }\end{array}$ \\
\hline
\end{tabular}

$\mathrm{Q}$ and $\mathrm{W}$ - larvae were developed to be queens and workers in laboratory.

Reconfirmation of caste differentiation by frozen sections. Results from frozen sections revealed that new adults from group Q had fully developed ovaries (Figure 4). They had significantly more ovarian tubes (290-360 each) toward the caste of worker bee than new adults from group W (20-30 each). Since each queen and worker of Apis mellifera ligustica has, on average, 300-400 and 10 ovarian tubes, respectively, the new adults from groups Q and W had the typical caste characteristics of the queen and worker, respectively. We concluded that water-soluble extracts in the larval diets inhibited the development of the ovary and induced larvae to develop toward the caste of worker bee.
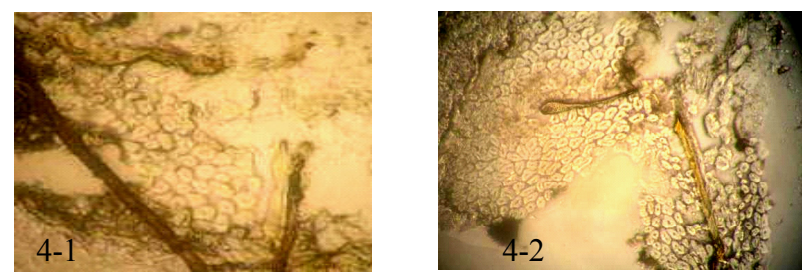

Figure 4. Frozen sections of ovary: 4-1 was one-side ovarian of new adults from larvae were developed to be workers; group W $(\times 4000)$ and 4-2 was bilateral ovaries of new adults from larvae were developed to be queens; group Q $(\times 1600)$

Screen differentially displayed $m R N A$. Differentially expressed amplicons were displayed on $12 \%$ polyacrylamide gel (Figure 5). A total of 67 transcriptderived fragments were cut off from the gels and subjected to re-amplification. Some fragments did not show positive amplification, which could be attributed to difficulties in gel band removal and DNA elution, or inhibition of PCR by some gel components. Those fragments were taken as false-positive fragments and discarded. Only those fragments that could produce amplicons with the same specific length as in the first amplification stage were used to produce recombinant plasmids and sequenced afterwards. 
After dot-blot hybridization, which was conducted to verify the fidelity of these differentially expressed fragments (Figure 6), we obtained a total of 6 differentially expressed fragments, designated as EST01-06, which were induced by water-soluble extracts from pollen during caste differentiation.

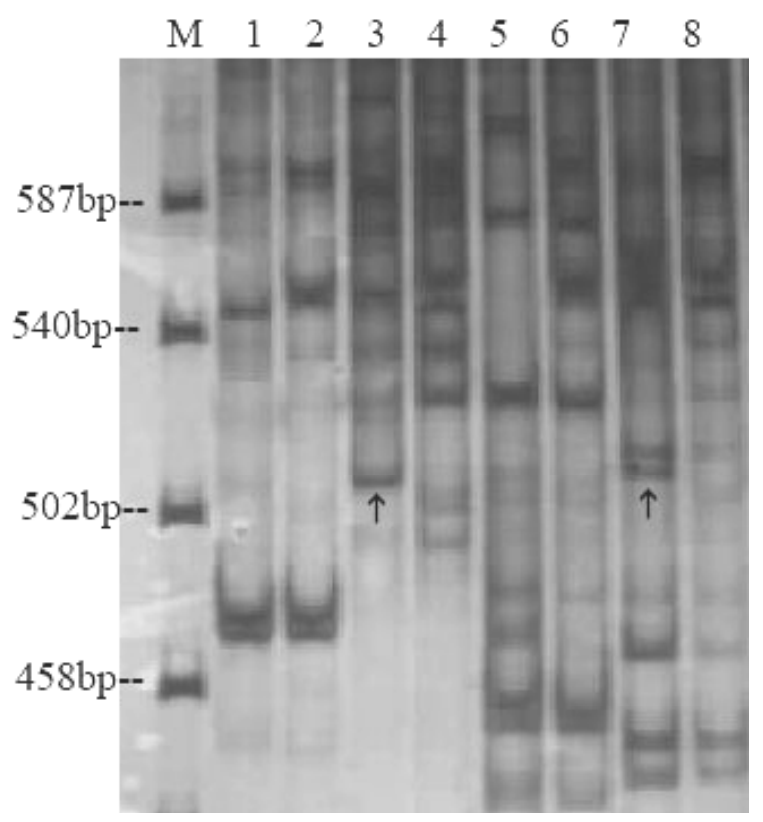

Figure 5. DDRT-PCR profile from 3-day old larvae. Lane 1, 3, 5 and 7 were amplicons from group W. Lane 2, 4, 6 and 8 were amplicons from group Q. The 3' single-anchored primer was 5'AAGCTTTTTTTTTTC -3' and the 5' 10-mer random primer was No.226 for lane 1 and lane 2, No. 234 for lane 3 and lane 4, No.235 for lane 5 and lane 6, No. 240 for lane 7 and lane 8, respectively. Lane M was loaded with the Marker, pBR322 / HaeIII. The differentially expressed amplicons were marked by arrows

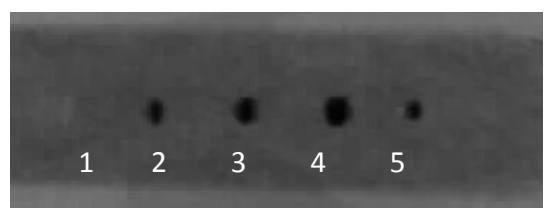

Figure 6. Dot-blot DNA-DNA hybridization using cDNA from total larval RNA as target and the digoxigen-labeled PCR products (EST-05) as the probe. 1. negative control; 2, 3. and 4. cDNA from 3-day old larvae in group Q with increasing concentrations; 5: positive control of the kit 
Nucleotide sequence alignment analysis. A homology search using nucleotide blast was performed to attribute a putative function to differentially expressed clones and among the 6 sequences analysed, 5 clones showed high similarity to genes from the honeybee with a general function (Table 4).

Table 4. Information of 6 differentially expressed fragments after BLAST

\begin{tabular}{lccccccc}
\hline Clone & Stage & Group & Size & \multicolumn{1}{c}{ Chromosome } & Gene & Similarity & E value \\
\hline EST-01 & 3d & W & 276bp & 15 (LOC552739) & GN366745 & $100 \%$ & $6 \mathrm{e}-140$ \\
EST-02 & 3d & W & 297bp & 1(LOC725608) & GN753584 & $100 \%$ & $3 \mathrm{e}-155$ \\
EST-03 & 3d & W & 340bp & - & - & - & - \\
EST-04 & 4d & W & 297bp & 13(CG9867) & CG9867-PA & $99 \%$ & $1 \mathrm{e}-153$ \\
EST-05 & 3d & Q & 236bp & 1(LOC550723) & - & $100 \%$ & $2 \mathrm{e}-121$ \\
EST-06 & 4d & Q & 531bp & 4(LOC724472) & KIAA0406 & $96 \%$ & 0.0 \\
\hline
\end{tabular}

Q and W - larvae will develop to be queens and workers, respectively

Clone EST-01 was similar to the farnesyltransferase (FTase) sequence from Apis mellifera. FTase is responsible for the addition of a farnesyl group to its target proteins bearing a CaaX motif at the carboxyl terminus. Modification by FTase is the premise for activation of the Ras superfamily, which includes small GTP-binding proteins involved in signal transduction of various biochemical and physiological processes. Increased activity of this enzyme could be required by the specialized metabolic pathways related to cell proliferation induced during caste differentiation. Its up-regulated expression in 3-d-old larvae from group W may correspond to the cascaded amplification induced by water-soluble extracts from pollen.

An internal regulatory factor from the RhoGAPs (Rho GTPase-activating proteins) superfamily, RhoGAP88c, showed the highest similarity to clone EST02. RhoGAPs are evolutionarily conserved proteins containing the RhoGAP domain. They interact with Rho GTPases to stimulate their intrinsic GTPase activity and work as molecular switches involved in the regulation of diverse cellular functions, including actin cytoskeleton rearrangements, regulation of gene transcriptions, cell cycle regulation, control of apoptosis, and membrane trafficking. The up-regulation of this gene in 3-d-old larvae from group $\mathrm{W}$ could be part of the regulatory mechanism of proliferation-related proteins in cells.

We did not find any similarity between clone EST-03 and any functional genes in the honeybee. No specific or functional properties could be inferred by the homology search. It may be a new EST. At present it is not possible to attribute a specific function to this gene or to draw any conclusions about the putative role of this gene in the differentiation of honeybee.

An interesting result of the differential analysis was the isolation of a transcript putatively related to glycosyltransferase, which was responsible for the transfer of 
a monosaccharide unit from an activated sugar phosphate to an acceptor molecule and to which clone EST-04 showed high similarity. Since many proteins undergo glycosyl modification before they become mature after translation, the presence of glycosyltransferase in 4-d-old larvae from group $\mathrm{W}$ confirmed the active synthesis of proteins in cells, which was inconsistent with the biochemical processes during the differential period.

Clone EST-05 showed high similarity to the Rab39 protein, which belongs to the Ras superfamily (a large number of small molecular weight GTPases). Rab GTPases are regulators of intracellular vesicular transport and the trafficking of proteins between different organelles of the endocytic and exocytic pathways (Zerial et al., 2001). Rab39 is associated with Golgi-related organelles and involved in the forming and improving of the immune system in humans.

A similarity to a KIAA0406 protein, which is closely involved in accelerated cell growth and division, was observed for clone EST-06. Up-regulated in 4-dold larvae from group $\mathrm{Q}$, the precise function of this protein could still not be unequivocally attributed.

It can be speculated that these differentially expressed genes encode proteins involved in the interactions of regulating signal networks, which are activated by nutrition-driven stimuli at early larval stages and are essential for the growth and function of differential pathways in the honeybee.

\section{DISCUSSION}

Nutrition, temperature and humidity are three important factors that play critical roles in the development of larvae. In this study, artificial larval diets contained about $69 \%$ water and $16 \%$ honey to stimulate larvae to take in food. The optimal temperature and relative humidity in the incubator were set according to our previous reports (Zhou et al., 2008). Results showed that the daily weight gain of larvae in two tested groups did not differ significantly from those developed under natural conditions, which confirmed that both the nutritional and environmental needs for the development of larvae were met in the incubator.

We determined the caste of new adults from three aspects: the entire developmental period (duration of the developmental period), external features, and the degree of development of the ovary. The results revealed that new adults from group $\mathrm{W}$ with pollen extracts in larval diets had typical caste features of worker bees, while new adults from group Q, developed from the same battery of fertilized eggs, became queen bees. Our results reconfirmed former conclusions that developmental fate was determined by nutritional regulation (Wheeler et al., 2006) and early intake of pollen at the larval stage triggered the development of 
worker bee morphology.

The quality of pollen and the protein:carbohydrate ratio were considered to be dietary factors determining reproductive capacity (Human et al., 2007). In this study we confirmed that extracts from pollen changed the developmental direction of larvae. We suggest that larvae in group Q were destined to become queens not because of the presence of royal jelly and honey in the diets but because of the absence of pollen. We conclude that pollen was the key substance that changed the direction of differentiation. Water-soluble extracts from pollen could offset the function of 'queen-determining substances' and reduce the taking-in activities of larvae, which resulted in the significantly lower larval weights in group $\mathrm{W}$. The exact relationship between components in water-soluble extracts from pollen and their functions still needs to be established.

To date, many studies on screening differentially expressed genes between queens and workers in the honeybee have been conducted. Some reports identified specific functional genes involved in caste development (Corona et al., 1999; Yamazaki et al., 2006; Lattorff et al., 2007; Aamodt 2008; de Azevedo et al., 2008). Others screened clusters of genes that belonged to distinctly different evolutionary and functional groups and pathways (Evans et al., 1999; Patel et al., 2007; Vieira et al., 2008). In this study, differential feeding of female larvae promotes the occurrence of two different phenotypes from identical genotypes. We screened six differentially expressed fragments with four of them up-regulated in prospective workers and three of them (EST-01, 02 and 03) were transcripts of newly discovered functional genes involved in caste differentiation (compared with the results of ArrayExpress accession number E-MEXP-1394). The results in our present study suggest that the worker's developmental pathway involved the participation of more novel genes, which is consistent with previous reports (Barchuk et al., 2007). It is still difficult to establish the precise role of these upregulated genes on the differentiation of the honeybee and the limited number of screened genes makes it difficult to apply cluster analysis at present. Compared with other techniques, DDRT-PCR presents a relatively high number of falsepositive clones. A large part of differentially expressed fragments were discarded in the re-amplification process and a confirmatory technique, dot-blot assay, was applied to reconfirm positive fragments representing differentially expressed genes, which gave rise to the relatively small number of positive clones.

The quality of pollen and the protein:carbohydrate ratio were considered to be dietary factors determining reproductive capacity (Human et al., 2007). In this study we confirmed that extracts from pollen changed the developmental direction 
of larvae. We suggest that larvae in group Q were destined to become queens not because of the presence of royal jelly and honey in the diets but because of the absence of pollen. It was pollen that changed the direction of differentiation. As we know, DNA methyltransferase (Dnmt) is involved in epigenetic regulation in Apis (Kucharski et al., 2008; Maleszka, 2008). Silencing the expression of Dnmt3 in newly hatched larvae led to a royal jelly-like effect on the larval developmental trajectory. It would be interesting to investigate whether this effect still exists in the presence of pollen-extracts. In our experiment, water-soluble extracts from pollen could offset the function of 'queen-determining substances' in royal jelly and reduce the taking-in activities of larvae, which resulted in the significantly lower larval weights in group W. Our results are compatible with the widely accepted notion that a high royal jelly intake by a feeding larva activates the biochemical and physiological pathways that destine it to be a queen. We focused more on the pollen-driven phenomenon of worker development and suggested that the "magic formula" responsible for the making of a worker, not a queen, is pollen. The detailed relationship between components in water-soluble extracts from pollen and their functions still needs to be established.

\section{CONCLUSIONS}

We proved that pollen in the diet was the key component of caste determination. Queens/workers responded to nutritional conditions by displaying phenotypic and reproductive plasticity, which was manifested by contrasting adult morphologies as well as different developmental degrees of the ovary. Six genes were differentially expressed as the result of the addition of water-soluble components from pollen to the larval diet, and were screened by DDRT-PCR analysis and confirmed by dotblot assay. In this study, three new members of caste differentiation-related genes were identified, which enriched our knowledge about the proximate molecular mechanisms underlying the process of caste differentiation in the honeybee.

\section{REFERENCES}

Aamodt R.M., 2008. The caste- and age-specific expression signature of honeybee heat shock genes shows an alternative splicing-dependent regulation of Hsp90. Mech. Ageing Dev. 129, 632637

Barchuk A.R., Cristino A.S., Kucharski R., Costa L.F., Simões Z.L., Maleszka R., 2007. Molecular determinants of caste differentiation in the highly eusocial honeybee Apis mellifera. BMC Develop. Biol. 18, 70 (Abstr.) 
Corona M., Estrada E., Zurita M., 1999. Differential expression of mitochondrial genes between queens and workers during caste determination in the honeybee, Apis mellifera. J. Exp. Biol. 202, 929-938

De Azevedo S. V., Hartfelder K., 2008. The insulin signaling pathway in honey bee (Apis mellifera) caste development - differential expression of insulin-like peptides and insulin receptors in queen and worker larvae. J. Insect Physiol. 54, 1064-1071

Evans J.D., Wheeler D.E., 1999. Differential gene expression between developing queens and workers in the honey bee, Apis mellifera. Proc. Nat. Acad. Sci. USA 96, 5575-5580

Evans J.D., Wheeler D.E., 2000. Expression profiles during honeybee caste determination. Genome Biol. 2, 1-6

Grozinger C.M., Fan Y., Hoover S.E., Winston M.L., 2007. Genome-wide analysis reveals differences in brain gene expression patterns associated with caste and reproductive status in honey bees (Apis mellifera). Mol. Ecol. 16, 4837-4848

Guidugli K.R., Hepperle C., Hartfelder K., 2004. A member of the short-chain dehydrogenase/ reductase (SDR) superfamily is a target of the ecdysone response in honey bee (Apis mellifera) caste development. Apidologie 35, 37-47

Hepperle C., Hartfelder K., 2001. Differentially expressed regulatory genes in honey bee caste development. Naturwissenschaften 88, 113-116

Human H., Nicolson S. W., Strauss K., Pirk C. W., Dietemann V., 2007. Influence of pollen quality on ovarian development in honeybee workers (Apis mellifera scutellata). J. Insect Physiol. 53, $649-655$

Jay D.E., Diana E.W., 2001. Expression profiles during honeybee caste determination. Genome Biol. 2(1): research 0001.1-research0001.6

Kucharski R., Maleszka J., Foret S., Maleszka R., 2008. Nutritional control of reproductive status in honeybees via DNA methylation. Science 319, 1827-1830

Lattorff H.M., Moritz R.F., Crewe R.M., Solignac M., 2007. Control of reproductive dominance by the thelytoky gene in honeybees. Biol. Lett. 3, 292-295

Maleszka R., 2008. Epigenetic interaction of environmental and genomic signals in honey bees. Epigenetics 3, 188-192

Mike H.A., Johan N.M.C., Willem J.B., 2003. Differential feeding of worker larvae affects caste characters in the Cape honeybee, Apis mellifera capensis. Behav. Ecol. Sociobiol. 54, 555 $-561$

Patel A., Fondrk M.K., Kaftanoglu O., Emore C., Hunt G., Frederick K., Amdam G.V., 2007. The making of a queen: TOR pathway is a key player in diphenic caste development. PLoS ONE, 2, e509

Piulachs M.D., Guidugli K.R., Barchuk A.R., Cruz J., Simões Z.L.P., Bellés X., 2003. The vitellogenin of the honey bee, Apis mellifera: structural analysis of the cDNA and expression studies. Insect Biochem. Molec. Biol. 33, 459-465

Vieira C.U., Bonetti A.M., Simões Z.L., Maranhão A.Q., Costa C.S., Costa M.C., Siquieroli A.C., Nunes F.M., 2008. Farnesoic acid O-methyl transferase (FAMeT) isoforms: conserved traits and gene expression patterns related to caste differentiation in the stingless bee, Melipona scutellaris. Arch. Insect Biochem. Physiol. 67, 97-106

Wheeler D. E., 1986. Developmental and physiological determinants of caste in social Hymenoptera: evolutionary implications. Amer. Naturalist 128,13-34

Wheeler D.E., Buck N., Evans J.D., 2006. Expression of insulin pathway genes during the period of caste determination in the honey bee, Apis mellifera. Insect Mol. Biol. 15, 597-602 
Yamazaki Y., Shirai K., Paul R. K., Fujiyuki T., Wakamoto A., Takeuchi H., Kubo T., 2006. Differential expression of HR38 in the mushroom bodies of the honeybee brain depends on the caste and division of labor. FEBS Lett. 15 580, 2667-2670

Zerial M., McBride H., 2001. Rab proteins as membrane organizers. Nat. Rev. Mol. Cell Biol. 2, 107-117

Zhou B., Ye M.H., Zhang K., 2008. Studies on the artificial feeding conditions of queen bee larvae.

J. Biol. Sci. 8, 950-953 